\title{
Complication of dilute vinegar therapy for external auditory canal cholesteatoma
}

\author{
Annabelle C. Leong • David A. Bowdler
}

Received: 1 April 2012/ Accepted: 20 April 2012/Published online: 11 May 2012

(C) Springer-Verlag 2012

\section{Dear Editor,}

We read with great interest the recent article by Chang et al. [1] which advocated the use of repeat irrigation with dilute vinegar in conjunction with regular microscopic local cleansing to manage spontaneous external auditory canal (EAC) cholesteatoma. In their series of 16 patients, they declared no complications resulting from this treatment regimen. However, we wish to report that when we trialled the very same regimen on one of our patients, our patient suffered a perforated tympanic membrane with subsequent worsened pain and otorrhoea, just 7 days after starting this treatment. Her external canal cholesteatoma was classified as grade I according to Chang's suggested temporal bone CT staging, i.e., with flattening of the bony external canal. She had surgical debridement and reconstruction of the EAC 6 months previously and was keen to explore another treatment option apart from repeat surgical intervention. She followed the self-cleansing instructions exactly as described in Chang's paper, using a 10-ml syringe to gently irrigate her ear canal with dilute vinegar, $40 \mathrm{ml}$ in total volume daily, aiming towards the posterior canal wall. When the tympanic membrane perforation was detected at her next clinic visit, she was instructed to stop the vinegar washouts and to commence a course of quinolone ear drops, after which her otorrhoea improved. She is currently still attending our clinic regularly for repeat microsuctioning of her canal cholesteatoma. If her perforated tympanic membrane fails to heal (and it has not done so thus far after 5 weeks), then performing a tympanoplasty would be the next obvious option.Cadaveric studies have found an association of ear irrigation with rupture of atrophic tympanic membranes with the lowest tensile strength but not of normal tympanic membranes [2]. Our patient's tympanic membrane was essentially normal before the perforation event and her cholesteatoma is solely confined to the EAC, as confirmed on computed tomography scanning on two separate occasions. We would like to politely enquire if the authors have experienced similar complications since publication of their paper or if they have any further insights into the vinegar regimen to share with us.

Thank you very much.

Yours sincerely, Annabelle C. Leong, FRCS (ORL-HNS)

David A. Bowdler, FRCS

\section{References}

1. Chang J, Choi C, Im GJ, Jung HH (2012) Dilute vinegar therapy for the management of spontaneous external auditory canal cholesteatoma. Eur Arch Otolrhinolaryngol 269:481-485

2. Sorensen VZ, Bonding P (1995) Can ear irrigation cause rupture of the tympanic membrane? An experimental study in man. J Laryngol Otol 109:1036-1040
A. C. Leong $(\bowtie) \cdot$ D. A. Bowdler

Department of Otolaryngology, University Hospital Lewisham,

London SE13 6LH, UK

e-mail: ackleong@gmail.com 